\title{
STRUCTURAL THEOREMS FOR PERIODIC ULTRADISTRIBUTIONS
}

\author{
STEVAN PILIPOVIĆ \\ ABSTRACT. Structural theorems for periodic ultradistributions of Roumieu and \\ Beurling types are given.
}

1. Introduction. Periodic ultradistributions spaces $\mathscr{D}^{\prime}\left\{m_{k}\right\}$ and $\mathscr{D}^{\prime}\left(m_{k}\right)$ of Roumieu type and Beurling type, respectively, are investigated in [1 and 2]. In the mentioned papers periodic ultradistributions are characterized by the growth rate of their coefficients. We notice that these spaces are subspaces of the space $\mathscr{P}^{\prime}$ of periodic generalized functions investigated in [5].

In this paper we shall give representation theorems for elements from $\mathscr{D}^{\prime}\left\{m_{k}\right\}$ and $\mathscr{D}^{\prime}\left(m_{k}\right)$.

2. Notations and preliminary results. In this part we shall recall basic notions from [2]. Also, we shall use notations from [2].

Sequence $m_{k}$ is a sequence of positive numbers for which in [2] it is supposed:

(1) $m_{k}^{2} \leqslant m_{k-1} m_{k+1}, k=1,2, \ldots$;

(2) $\sum_{k=1}^{\infty} m_{k-1} / m_{k}<\infty$;

(3) There are constants $A$ and $H$ such that $m_{k+1} \leqslant A H^{k} m_{k}, k=0,1, \ldots$

(4) There are constants $A$ and $H$ such that

$$
m_{k} \leqslant A H^{k} \min _{0 \leqslant q \leqslant k} m_{q} m_{k-q}, \quad k=0,1, \ldots
$$

We note that these conditions and some others are analyzed in [3] in detail. If $s>1$, the Gevrey sequence $m_{k}=(k !)^{s}$ or $k^{k s}$ or $\Gamma(1+k s)$ satisfies the above conditions.

Condition (1) directly implies

$$
m_{q} m_{k} \leqslant m_{0} m_{k+q}, \quad q=0,1, \ldots, k=0,1, \ldots
$$

Thus condition (4) from [2] is superfluous.

Conditions (1)-(4) imply "good" properties of periodic ultradistributions spaces (see [2]). For our investigations we shall assume that the sequence $m_{k}$ satisfies conditions (1), (3) and instead of (2) we shall assume

$(2)^{*} \lim _{k \rightarrow \infty} \sqrt[k]{m_{k}}=\infty$

Received by the editors October 26, 1984 and, in revised form, September 12, 1985.

1980 Mathematics Subject Classification (1985 Revision). Primary Primary 46Fxx, 46F12.

Key words and phrases. Periodic ultradistributions of Roumieu and Beurling type.

This material is based on work supported by the U.S.-Yugoslav Joint Fund for Scientific and Technological Cooperation, in cooperation with the (JFP) under Grant 544. 
Since $m_{k}=m_{0}\left(m_{1} / m_{0}\right) \cdots\left(m_{k} / m_{k-1}\right),(2)$ implies (2)*. If $m_{k}=(k !)^{\alpha}, 0<\alpha$ $\leqslant 1$, then (2)* holds but (2) does not.

One can easily prove that (2)* is equivalent to the fact that the space $\mathscr{P}$ of polynomials of the form $\sum_{k=-n}^{n} c_{k} e_{k}(t), c_{k}$ are complex numbers and $e_{k}=\exp (i k t)$, $k=0, \pm 1, \ldots$, is a subspace of $\mathscr{D}\left(m_{k}\right)$ and therefore of $\mathscr{D}\left\{m_{k}\right\}$ (see below).

As in [2] we denote by $\mathscr{D}\left(m_{k}, L\right), L>0$, the space of all smooth functions on the unit circle $K$, such that $\phi \in \mathscr{D}\left(m_{k}, L\right)$ iff

$$
\begin{aligned}
\|\phi\|_{L, \infty}:=\sup \left\{\frac{\left\|\phi^{(k)}\right\|_{\infty}}{L^{k} m_{k}} ; k=0,1, \ldots\right\} & <\infty \\
& \left(\|\phi\|_{\infty}=\sup \{|\phi(t)| ; t \in[0,2 \pi]\}\right) .
\end{aligned}
$$

The space $\mathscr{D}\left(m_{k}, L\right)$ is a $(B)$-space. Spaces $\mathscr{D}\left\{m_{k}\right\}$ and $\mathscr{D}\left(m_{k}\right)$ are defined as

$$
\mathscr{D}\left\{m_{k}\right\}=\text { ind } \lim _{L \rightarrow \infty} \mathscr{D}\left(m_{k}, L\right), \quad \mathscr{D}\left(m_{k}\right)=\text { proj } \lim _{L \rightarrow 0} \mathscr{D}\left(m_{k}, L\right),
$$

where we take these limits in the topological sense. In the sense of strong topologies in $\mathscr{D}^{\prime}\left\{m_{k}\right\}$ and $\mathscr{D}^{\prime}\left(m_{k}\right)$ we have [2],

$$
\mathscr{D}^{\prime}\left\{m_{k}\right\}=\text { proj } \lim _{L \rightarrow \infty} \mathscr{D}^{\prime}\left(m_{k}, L\right), \quad \mathscr{D}^{\prime}\left(m_{k}\right)=\text { ind } \lim _{L \rightarrow 0} \mathscr{D}^{\prime}\left(m_{k}, L\right) .
$$

In [6, Chapter 9], Zemanian investigated spaces $\mathscr{A}$ and $\mathscr{A}^{\prime}$. As a particular case he investigated spaces $\mathscr{A}$ and $\mathscr{A}^{\prime}$ which correspond to the space $L^{2}(0,2 \pi)$ and the differential operator $\mathscr{R}=i D$ ( $D$ is the derivative). Let us denote these spaces by $\mathscr{A}_{\text {per }}$ and $\mathscr{A}_{\text {per }}^{\prime}$. We remark that these spaces may be identified with the spaces $\mathscr{D}$ and $\mathscr{D}^{\prime}$ (for the definition of the spaces $\mathscr{D}$ and $\mathscr{D}^{\prime}$ see [1]).

If $\phi$ belongs to $\mathscr{D}\left\{m_{k}\right\}$ or $\mathscr{D}\left(m_{k}\right)$ its Fourier coefficients

$$
c_{k}(\phi)=\left\langle\phi, e_{k}(-t)\right\rangle=\int_{0}^{2 \pi} \phi(t) e_{k}(-t) d t, \quad k=0, \pm 1, \ldots,
$$

decrease more rapidly than any power of $|k|$ when $|k| \rightarrow \infty[2, \mathrm{p}$. 146]. This implies that $\mathscr{D}\left\{m_{k}\right\}$ and $\mathscr{D}\left(m_{k}\right)$ are subspaces of $\mathscr{A}_{\text {per }}$ (see [6, Theorem 9.3.3]). The space $\mathscr{A}_{\text {per }}$ consists of elements from $L^{2}(0,2 \pi) \cap C^{\infty}(0,2 \pi)$ for which

$$
\begin{aligned}
\gamma_{p}(\phi):=\sup \left\{\left\|D^{i} \phi\right\|_{2} ; i \leqslant p\right\}<\infty, \quad p & =1,2, \ldots \\
& \left(\|\phi\|_{2}=\left(\int_{0}^{2 \pi}|\phi(t)|^{2} d t\right)^{1 / 2}\right)
\end{aligned}
$$

and

$$
\left\langle D^{k} \phi, e_{n}(t)\right\rangle=(-i n)^{k}\left\langle\phi, e_{n}(t)\right\rangle, \quad k=0,1, \ldots, n=0, \pm 1, \ldots
$$

THEOREM 1. $\mathscr{D}\left\{m_{k}\right\}$ and $\mathscr{D}\left(m_{k}\right)$ can be identified with $\mathscr{A}_{\text {per }}\left\{m_{k}\right\}$ and $\mathscr{A}_{\text {per }}\left(m_{k}\right)$ respectively, where

$$
\mathscr{A}_{\mathrm{per}}\left\{m_{k}\right\}=\text { ind } \lim _{L \rightarrow \infty} \mathscr{A}_{\mathrm{per}}\left(m_{k}, L\right), \quad \mathscr{A}_{\mathrm{per}}\left(m_{k}\right)=\operatorname{prod} \lim _{L \rightarrow 0} \mathscr{A}_{\mathrm{per}}\left(m_{k}, L\right),
$$

and

$$
\mathscr{A}_{\mathrm{per}}\left(m_{k}, L\right)=\left\{\phi \in \mathscr{A}_{\mathrm{per}} ;\|\phi\|_{L, 2}:=\sum_{k=0}^{\infty} \frac{\left\|\phi^{(k)}\right\|_{2}}{L^{k} m_{k}}<\infty\right\} .
$$


Proof. If $\phi \in \mathscr{D}\left(m_{k}, L / 2\right)$, then $\phi \in \mathscr{A}_{\text {per }}\left(m_{k}, L\right)$. Let $\phi(t)=\sum_{k=-\infty}^{\infty} a_{k} e_{k}(t) \in$ $\mathscr{A}_{\text {per }}\left(m_{k}, L\right)$. This series converges uniformly to $\phi$ on $[0,2 \pi]$. The same holds for

$$
\phi^{\prime}(t)=\sum_{k=-\infty}^{\infty} a_{k}(i k) e_{k}(t), \quad t \in[0,2 \pi]
$$

We have

$$
\|\phi\|_{\infty} \leqslant \sum_{k=-\infty}^{\infty}\left|a_{k}\right| \leqslant\left(\sum_{k=-\infty}^{\infty}\left|a_{k}\right|^{2} k^{2}\right)^{1 / 2}\left(\sum_{k=-\infty}^{\infty} k^{-2}\right)^{1 / 2}=C\left\|\phi^{\prime}\right\|_{2}
$$

where $C=2 \sum_{k=0}^{\infty} k^{-2}$. Using (3) we obtain

$$
\frac{\|\phi\|_{\infty}}{L^{k} m_{k}} \leqslant \frac{A C}{H} \frac{\left\|\phi^{\prime}\right\|_{2}}{(L / H)^{k+1} m_{k+1}} .
$$

Thus if $\phi \in \mathscr{A}_{\text {per }}\left(m_{k}, L / H\right)$ then $\phi \in \mathscr{D}\left(m_{k}, L\right)$. It is easy now to prove the assertion of Theorem 1 .

\section{Representation theorems.}

TheOREM 2. If $f \in \mathscr{D}^{\prime}\left(m_{k}\right)$ then there exist a sequence of functions $f_{i}, i=0,1, \ldots$, on $(0,2 \pi)$ and a natural number $n$ such that

(i) $f_{i} \in L^{2}(0,2 \pi), i=0,1, \ldots$;

(ii) $\sup _{i}\left\|f_{i}\right\|_{2}<\infty$;

(iii) $f(t)=\sum_{i=0}^{\infty}\left(n^{i} / m_{i}\right) f_{i}^{(i)}(t)$.

Conversely, if $f_{i}, i=0,1, \ldots$, is a sequence of functions on $(0,2 \pi)$ for which (i) and (ii) hold, then by the series on the right side of (iii) a unique element from $\mathscr{D}^{\prime}\left(m_{k}\right)$ is defined.

The convergence of the series in (iii) is understood in a weak sense.

In the proof of Theorem 2 we shall show that $\mathscr{D}\left(m_{k}\right)$ is a projective limit of a reduced compact sequence of $(B)$-spaces. Thus $\mathscr{D}\left(m_{k}\right)$ and $\mathscr{D}^{\prime}\left(m_{k}\right)$ are Montel spaces and the weak and strong sequential convergence in $\mathscr{D}^{\prime}\left(m_{k}\right)$ are coincident.

Proof. We denote by $\mathscr{A}_{\text {per }}\left(m_{k}, 1 / n\right)$ the subspace of $\mathscr{A}_{\text {per }}$ such that

$$
\phi \in \mathscr{A}_{\text {per }}\left(m_{k}, 1 / n\right) \text { iff }\|\phi\|_{1 / n, 2}<\infty,
$$

where $n$ is a fixed natural number.

Let $\phi_{\nu}$ be a Cauchy sequence in $\mathscr{A}_{\text {per }}\left(m_{k}, 1 / n\right)$. Since $\mathscr{A}_{\text {per }}$ is complete there is a $\phi_{0} \in \mathscr{A}_{\text {per }}$ such that (by Fatou's Lemma)

$$
\begin{aligned}
\sum_{k=0}^{\infty} \frac{n^{k}\left\|\phi_{\nu}^{(k)}-\phi_{0}^{(k)}\right\|_{2}}{m_{k}} & =\sum_{k=0}^{\infty} \frac{n^{k}}{m_{k}} \lim _{\mu \rightarrow \infty}\left\|\phi_{\nu}^{(k)}-\phi_{\mu}^{(k)}\right\|_{2} \\
& \leqslant \liminf _{\mu} \sum_{k=0}^{\infty} \frac{n^{k}}{m_{k}}\left\|\phi_{\nu}^{(k)}-\phi_{\mu}^{(k)}\right\|_{2} .
\end{aligned}
$$

This inequality implies that $\mathscr{A}_{\text {per }}\left(m_{k}, 1 / n\right)$ is complete. If $\phi \in \mathscr{A}_{\text {per }}\left(m_{k}, 1 / n\right)$ and $\phi(t)=\sum_{k=-\infty}^{\infty} c_{k} e_{k}(t)$, then the sequence $\sum_{k=-n}^{n} c_{k} e_{k}(t), n=0,1, \ldots$, converges to $\phi$ in the norm \|\|$_{1 / n, 2}$. Since for every $n \sum_{k=-n}^{n} c_{k} e_{k}(t) \in \mathscr{A}_{\text {per }}\left(m_{k}\right)$ it follows that $\mathscr{A}_{\text {per }}\left(m_{k}, 1 / n\right)$ is the completion of $\mathscr{A}_{\text {per }}\left(m_{k}\right)$ in the sense of the norm $\|.\|_{1 / n, 2}$. 
The sequence of norms \|\|$_{1 / n, \infty}, n=1,2, \ldots$, is equivalent with the sequence of norms \|\|$_{1 / n, 2}$ on $\mathscr{A}_{\mathrm{per}}\left(m_{k}\right)$. If we denote by $\overline{\mathscr{A}}_{\mathrm{per}}\left(m_{k}, 1 / n\right)$ the completion of $\mathscr{A}_{\text {per }}\left(m_{k}\right)$ in the sense of the norm \|\|$_{1 / n, \infty}, \overline{\mathscr{A}}_{\text {per }}\left(m_{k}, 1 / n\right)$ is identical with the space $\mathscr{D}\left(m_{k}, 1 / n\right)$.

From Theorem 1 it follows that for any $n_{0}$ there exist $n_{1}, n_{2}$, and $n_{3}, n_{0}<n_{1}<$ $n_{2}<n_{3}$, such that the inclusion mappings

$$
\mathscr{A}_{\text {per }}\left(m_{k}, 1 / n_{3}\right) \rightarrow \overline{\mathscr{A}}_{\mathrm{per}}\left(m_{k}, 1 / n_{2}\right) \rightarrow \overline{\mathscr{A}}_{\mathrm{per}}\left(m_{k}, 1 / n_{1}\right) \rightarrow \mathscr{A}_{\mathrm{per}}\left(m_{k}, 1 / n_{0}\right)
$$

are continuous. By the Ascoli-Arcela theorem we obtain that the mapping $\overline{\mathscr{A}}_{\text {per }}\left(m_{k}, 1 / n_{2}\right) \rightarrow \overline{\mathscr{A}}_{\text {per }}\left(m_{k}, 1 / n_{1}\right)$ is compact. It implies that the mapping $\mathscr{A}_{\text {per }}\left(m_{k}, 1 / n_{3}\right) \rightarrow \mathscr{A}_{\text {per }}\left(m_{k}, 1 / n_{0}\right)$ is compact as well. Thus we obtain (see [3, p. 33])

$$
\mathscr{D}^{\prime}\left(m_{k}\right) \equiv \mathscr{A}_{\mathrm{per}}^{\prime}\left(m_{k}\right)=\left(\operatorname{proj} \lim _{n \rightarrow \infty} \mathscr{A}_{\mathrm{per}}\left(m_{k}, \frac{1}{n}\right)\right)^{\prime}=\text { ind } \lim _{n \rightarrow \infty} \mathscr{A}_{\mathrm{per}}^{\prime}\left(m_{k}, \frac{1}{n}\right),
$$

in the sense of strong topologies in these spaces. It means that if $f \in \mathscr{D}^{\prime}\left(m_{k}\right)$ then $f$ can be uniquely extended on some $\mathscr{A}_{\text {per }}\left(m_{k}, 1 / n\right)$ such that $f \in \mathscr{A}_{\text {per }}^{\prime}\left(m_{k}, 1 / n\right)$. Conversely, if $f \in \mathscr{A}_{\text {per }}^{\prime}\left(m_{k}, 1 / n\right)$ then $f \in \mathscr{A}_{\text {per }}^{\prime}\left(m_{k}\right)$. Thus, it is enough to give a representation theorem for elements from $\mathscr{A}_{\text {per }}^{\prime}\left(m_{k}, 1 / n\right)$ for a fixed $n$. This can be done in the same way as in [4, proof of Theorem 6]; see also [3].

The space $\mathscr{A}_{\text {per }}\left(m_{k}, 1 / n\right)$ is isometric to the closed subspace $\Lambda$ of the space $\left(\Gamma,\|\|_{\Gamma}\right)$ where $\Gamma$ is the space of sequences $\left\{\psi_{i}\right\} \in \prod_{i=0}^{\infty} L^{2}(0,2 \pi)$ such that

$$
\left\|\left\{\psi_{i}\right\}\right\|_{\Gamma}:=\sum_{i=0}^{\infty}\left\|\psi_{i}\right\|_{2}<\infty
$$

This isometry is given by the mapping

$$
\mathscr{A}_{\mathrm{per}}\left(m_{k}, \frac{1}{n}\right) \ni \phi \mapsto\left\{\frac{(-1)^{i} n^{i} \phi^{(i)}}{m_{i}}\right\} \in \Lambda .
$$

Clearly $f$ defines an element from $\Lambda^{\prime}$ which we shall denote by $f_{1}$. By the Hahn Banach theorem we extend $f_{1}$ from $\Lambda$ to $\Gamma$ (we denote this extension by $F$ ) such that $F \in \Gamma^{\prime}$. Since $F \in \Gamma^{\prime}$, there exists a sequence $f_{i}, i=0,1, \ldots$, from $L^{2}(0,2 \pi)$ such that

$$
\left\langle F,\left\{\psi_{i}\right\}\right\rangle=\sum_{i=0}^{\infty}\left\langle f_{i}, \psi_{i}\right\rangle=\sum_{i=0}^{\infty} \int_{0}^{2 \pi} f_{i}(t) \psi_{i}(t) d t, \quad\left\{\psi_{i}\right\} \in \Gamma,
$$

and $\sup _{i}\left\|f_{i}\right\|_{2}<\infty$. If $\phi \in \mathscr{A}_{\text {per }}\left(m_{k}, 1 / n\right)$ we have

$$
\begin{aligned}
\langle f, \phi\rangle & =\left\langle f_{1},\left\{(-1)^{i} \frac{n^{i}}{m_{i}} \phi^{(i)}\right\}\right\rangle=\left\langle F,\left\{(-1)^{i} \frac{n^{i}}{m_{i}} \phi^{(i)}\right\}\right\rangle \\
& =\left\langle\sum_{i=0}^{\infty}\left(\frac{n^{i}}{m_{i}} f_{i}\right)^{(i)}, \phi\right\rangle .
\end{aligned}
$$

This completes the proof of the first part of Theorem 2.

The second part of Theorem 2 trivially holds because any function $F$ from $L^{2}(0,2 \pi)$ defines an element from $\mathscr{D}^{\prime}\left(m_{k}\right)$ :

$$
\phi \mapsto \int_{0}^{2 \pi} F(t) \phi(t) d t, \quad \phi \in \mathscr{D}\left(m_{k}\right) .
$$


THEOREM 3. If $f \in \mathscr{D}^{\prime}\left\{m_{k}\right\}$ then there exists a sequence of functions $f_{i}, i=0,1, \ldots$, on $(0,2 \pi)$ such that

(i) $f_{i} \in L^{2}(0,2 \pi)$;

(ii) $\sum_{i=0}^{\infty}\left\|n^{i} m_{i} f_{i}\right\|_{2}<\infty$, for every natural number $n$;

(iii) $f(t)=\sum_{i=0}^{\infty} f_{i}^{(i)}(t)$.

Conversely, if $f_{i}$ is a sequence of functions on $(0,2 \pi)$ such that (i) and (ii) hold, then with the series on the right side of (iii) a unique element from $\mathscr{D}^{\prime}\left\{m_{k}\right\}$ is defined.

The convergence of the series in (iii) is understood in the weak sense.

In the proof which follows, we shall show that $\mathscr{A}_{\text {per }}\left\{m_{k}\right\}$ is an inductive limit of an injective compact sequence of $(B)$-spaces. It implies that $\mathscr{D}\left\{m_{k}\right\}$ and $\mathscr{D}^{\prime}\left\{m_{k}\right\}$ are Montel spaces. So the weak and strong sequential convergence in $\mathscr{D}^{\prime}\left\{m_{k}\right\}$ are coincident.

Proof. We denote by $\mathscr{A}_{\text {per }}\left(m_{k}, n\right)$, where $n$ is a fixed natural number, a subset of $\mathscr{A}_{\text {per }}$ such that $\phi \in \mathscr{A}_{\text {per }}\left(m_{k}, n\right)$ iff $\|\phi\|_{n, 2}<\infty$.

Obviously, this space is a $(B)$-space. As in the proof of Theorem 2, for any $n_{0}$ one can find $n_{3}$ such that the inclusion mapping $\mathscr{A}_{\mathrm{per}}\left(m_{k}, n_{0}\right) \rightarrow \mathscr{A}_{\mathrm{per}}\left(m_{k}, n_{3}\right)$ is compact. Thus we have [3, p. 33]

$$
\begin{aligned}
\mathscr{D}^{\prime}\left\{m_{k}\right\} & \equiv \mathscr{A}_{\text {per }}^{\prime}\left\{m_{k}\right\}=\left(\text { ind } \lim _{n \rightarrow \infty} \mathscr{A}_{\text {per }}\left(m_{k}, n\right)\right)^{\prime} \\
& =\text { proj } \lim _{n \rightarrow \infty} \mathscr{A}_{\text {per }}^{\prime}\left(m_{k}, n\right) .
\end{aligned}
$$

We denote by $Y_{n}$, where $n$ is a fixed number, the space of sequences $\left\{\psi_{i}\right\}$, $\psi_{i} \in L^{2}(0,2 \pi), i=0,1, \ldots$, such that

$$
\left\{\psi_{i}\right\} \in Y_{n} \text { iff }\left\|\left\{\psi_{i}\right\}\right\|_{Y_{n}}:=\sum_{i=0}^{\infty} \frac{\left\|\phi_{i}\right\|_{2}}{n^{i} m_{i}} .
$$

Spaces $Y_{n}, n=1,2, \ldots$, are $(B)$-spaces. $\mathscr{A}_{\mathrm{per}}\left(m_{k}, n\right)$ is isometric with the closed subspace $\Lambda$ of $Y_{n}$. This isometry is given by the mapping $\mathscr{A}_{\text {per }}\left(m_{k}, n\right) \in \phi \mapsto$ $\left\{(-1)^{i} \phi^{(i)}\right\} \in \Lambda$.

We denote $\mathscr{A}_{\text {per }}\left(m_{k}, n\right)$ by $X_{n}, n=1,2, \ldots$ In the same way as in [3, proof of Proposition 8.6] we prove that $Y_{n}$ is an injective weakly compact sequence. Since $X_{n}$ is an injective compact sequence, $Z_{n}=Y_{n} / X_{n}$ is also an injective sequence of (B)-spaces. Thus, it follows from the dual Mittag-Leffler lemma [3, p. 37] that if $f \in\left(\text { ind } \lim _{n \rightarrow \infty} \mathscr{A}_{\text {per }}\left(m_{k}, n\right)\right)^{\prime}$ then there exists $F \in\left(\text { ind } \lim _{n \rightarrow \infty} Y_{n}\right)^{\prime}$ such that on $\Lambda\langle f, \phi\rangle=\left\langle F,\left\{(-1)^{i} \phi^{(i)}\right\}\right\rangle$, and the mapping $f \mapsto F$ is continuous under the strong topologies in $\mathscr{D}^{\prime}\left\{m_{k}\right\}$ and (ind $\left.\lim _{n \rightarrow \infty} Y_{n}\right)^{\prime}$.

Since (ind $\left.\lim _{n \rightarrow \infty} Y_{n}\right)^{\prime}=$ proj $\lim _{n \rightarrow \infty} Y_{n}^{\prime}$ and this is the space of all sequences $f_{i}$, $f_{i} \in L^{2}(0,2 \pi), i=0,1, \ldots$, such that for every natural number $n \sum_{i=0} n^{i} m_{i}\left\|f_{i}\right\|_{2}<$ $\infty$, we obtain that this holds for $F$.

If $\phi \in \mathscr{A}_{\text {per }}\left\{m_{k}\right\}$ we have

$$
\langle f, \phi\rangle=\sum_{i=0}^{\infty}\left\langle f_{i},(-1)^{i} \phi^{(i)}\right\rangle=\left\langle\sum_{i=0}^{\infty} f_{i}^{(i)}(t), \phi(t)\right\rangle
$$


or

$$
f=\sum_{i=0}^{\infty} f_{i}^{(i)} \quad \text { in } \mathscr{D}^{\prime}\left\{m_{k}\right\} .
$$

Thus we proved the first part of Theorem 3 .

The strong topology in $\mathscr{D}^{\prime}\left\{m_{k}\right\}$ is determined by the family of seminorms

$$
p_{n}(f)=\sup \left\{|\langle f, \phi\rangle| ;\|\phi\|_{n, 2}<1\right\}, \quad n=1,2, \ldots
$$

If $\phi$ belongs to the unit ball in $\mathscr{A}_{\text {per }}\left(m_{k}, n\right)$ then

$$
\begin{aligned}
|\langle f, \phi\rangle| & =\left|\sum_{i=0}^{\infty}\left\langle f_{i},(-1)^{i} \phi^{(i)}\right\rangle\right| \leqslant \sum_{i=0}^{\infty} \int_{0}^{2 \pi}\left|f_{i} \phi^{(i)}\right| \\
& \leqslant \sum_{i=0}^{\infty}\left\|n^{i} m_{i} f_{i}\right\|_{2}\|\phi\|_{n, 2} \leqslant C\|\phi\|_{n, 2} .
\end{aligned}
$$

It implies that $p_{m}(f) \leqslant C$ so by the series in (iii) a unique element of $\mathscr{D}^{\prime}\left\{m_{k}\right\}$ is defined.

Let us remark that in the case $m_{k}=k$ ! the proofs of Theorems 2 and 3 are trivial. ADDED IN PROOF. An excellent reference is H.-J. Petzsche, Die Nuklearität der Ultradistributionsräume und der Satz vom Kern I, Manuscripta Math. 24 (1978), 133-171.

\section{REFERENCES}

1. V. I. Gorbačuk and M. L. Gorbačuk, Trigonometric series and generalized functions, Dokl. Akad. Nauk SSSR (4) 257 (1981), 799-804. (Russian)

2. V. I. Gorbacuk, On Fourier series of periodic ultradistributions, Ukranian Math. J. (2) 34 (1982), 144-150. (Russian)

3. H. Komatsu, Ultradistributions. I: Structure theorems and a characterizations, J. Fac. Sci. Univ. Tokyo Sect. I A 20 (1973), 25-105.

4. S. Pilipovic, Generalization of Zemanian space of generalized fucntions which have orthonormal expansion, SIAM J. Math. Anal. 17 (1986), 477-484.

5. A. Száz, Periodic generalized functions, Publ. Math. (Debrecen) 25 (1978), 227-235.

6. A. H. Zemanian, Generalized integral transformations, Interscience, New York, 1968.

INSTitute of Mathematics, UNiversity of Novi SAD, Novi SAD, Yugoslavia 